\title{
A public data set of human balance evaluations
}

\author{
Damiana A Santos ${ }^{1}$, Marcos Duarte ${ }^{\text {Corresp. } 1}$ \\ ${ }^{1}$ Biomedical Engineering, Universidade Federal do ABC, São Bernardo do Campo, SP, Brazil \\ Corresponding Author: Marcos Duarte \\ Email address: duartexyz@gmail.com
}

The goal of this study was to create a public data set with results of qualitative and quantitative evaluations related to human balance. Subject's balance was evaluated by posturography using a force platform and by the Mini Balance Evaluation Systems Tests. In the posturography test, we evaluated subjects standing still for $60 \mathrm{~s}$ in four different conditions where vision and the standing surface were manipulated: on a rigid surface with eyes open; on a rigid surface with eyes closed; on an unstable surface with eyes open; on an unstable surface with eyes closed. Each condition was performed three times and the order of the conditions was randomized. In addition, the following tests were employed in order to better characterize each subject: Short Falls Efficacy Scale International; International Physical Activity Questionnaire Short Version; and Trail Making Test. The subjects were also interviewed to collect information about their socio-cultural, demographic, and health characteristics. The data set comprises signals from the force platform (raw data for the force, moments of forces, and centers of pressure) of 163 subjects plus one file with information about the subjects and balance conditions and the results of the other evaluations. All the data is available at PhysioNet and at Figshare . 
1

2 A public data set of human balance evaluations

3

4

5 Damiana Aparecida dos Santos, Marcos Duarte

6

7 Biomedical Engineering, Universidade Federal do ABC, São Bernardo do Campo, SP, Brasil

8

9

10 Corresponding Author:

11 Marcos Duarte

12 duartexyz@gmail.com

13 


\section{Abstract}

15

16 The goal of this study was to create a public data set with results of qualitative and quantitative

17 evaluations related to human balance. Subject's balance was evaluated by posturography using a force platform and by the Mini Balance Evaluation Systems Tests. In the posturography test, we evaluated subjects standing still for $60 \mathrm{~s}$ in four different conditions where vision and the standing surface were manipulated: on a rigid surface with eyes open; on a rigid surface with eyes closed; on an unstable surface with eyes open; on an unstable surface with eyes closed.

22 Each condition was performed three times and the order of the conditions was randomized. In addition, the following tests were employed in order to better characterize each subject: Short Falls Efficacy Scale International; International Physical Activity Questionnaire Short Version; and Trail Making Test. The subjects were also interviewed to collect information about their socio-cultural, demographic, and health characteristics. The data set comprises signals from the

27 force platform (raw data for the force, moments of forces, and centers of pressure) of 163 subjects plus one file with information about the subjects and balance conditions and the results 29 of the other evaluations. All the data is available at PhysioNet and at Figshare. 
31

32

33

\section{Introduction}

Age-related disabilities and certain illnesses affect balance in humans and can negatively influence their health and quality of life. There has been great effort by researchers and clinicians for a greater understanding of this problem and postural control in humans has been under intense scientific investigation over the past decades.

There is a large variety of tests to describe balance in humans (for a review see Duarte \& Freitas 2010; Paillard \& Noe 2015; Scoppa et al. 2013; Shumway-Cook \& Woollacott 2001; Visser et al. 2008; Yamamoto et al. 2015). The most common quantitative measurement to characterize body balance is the displacement of the center of pressure (COP); the point of application of the resultant vertical forces acting on the subject's surface of support. COP displacement is typically measured with a force plate and presented as time-series of numerical data in the anterior-posterior (ap) and medio-lateral (ml) directions in relation to the subject's orientation. The technique concerned with the measurement of COP displacement in this context is commonly referred to as stabilography or posturography.

In stabilography, there is no consensus yet on the best techniques to analyze COP displacement in order to extract meaningful information about the subject's balance. There are also numerous different protocols (which include the type of task the subject performs, instructions to the subject, duration of the task, type of instrument, etc.) for data collection, algorithms, and variables to process and characterize COP displacement (for a review on these issues see Duarte \& Freitas 2010; Paillard \& Noe 2015; Ruhe et al. 2010; Scoppa et al. 2013; Visser et al. 2008; Yamamoto et al. 2015). Part of the lack of consensus and conflicting findings might be because of the typically large intra and inter-subject variability of the COP displacement data, and that some of the studies present low statistical power (typically because of a small sample size) (Ruhe et al. 2010; Samson \& Crowe 1996). A related problem, not 
55 specific to the field of stabilography, is that researchers propose and compare new methods of

56

57

58

59

60

61

62

63

64

65

66

67

analysis based on data from different subjects across centers.

The deployment of a public data set of human balance evaluations would allow the access of a normative reference for data comparison and testing analysis from different centers. In the human movement science field there are few publicly available data sets (for example, see Moore et al. 2015 and the references therein). However, none of the available data sets are about human balance. Hence, the purpose of the present study is to describe how a publicly available of balance evaluations on young and elderly adults was created.

\section{Methods}

This study was designed to create a public repository of data related to human balance, employing quantitative and qualitative evaluations. The entire data collection for each subject was performed in a single session, which lasted between one and two hours. Each subject was assessed by the same experienced examiner (D.A.S.) in the Laboratory of Biomechanics and Motor Control at the Federal University of ABC, Brazil. Prior to the evaluations that generated this data set, we conducted pilot studies with five subjects for training with the equipment and experimental protocol. The data of these subjects are not included in this data set. This study was approved by the local ethics committee of the Federal University of ABC (\#842529/2014).

\subsection{Subjects}

We evaluated a convenience sample of 163 subjects (116 females and 47 males) who voluntarily participated in this study. The subjects were recruited via flyers, advertising on social networks and word to mouth from local communities and included students, professors, and technicians from the university, the local neighborhood, and a community center for older adults. 
79 The subjects were first interviewed to collect information about their socio-cultural,

80 demographic, and health characteristics. Their ages varied from 18 to 85 years, body masses

81 from 44.0 to $75.9 \mathrm{~kg}$, heights from 140.0 to $189.8 \mathrm{~cm}$, and body-mass indexes (BMI) from 17.2

82 to $31.9 \mathrm{~kg} / \mathrm{m}^{2}$. Of the 163 subjects, 16 of them were classified as having at least one severe

83 disability or more (eight with hearing and vestibular deficits; two with visual deficits; three with

84 musculoskeletal deficits, one with visual and musculoskeletal deficits, one with hearing and

85 visual deficits, and one with intellectual disability). All this information for each subject are

86 presented in the public data set (see later on how to obtain it).

\subsection{Stabilography}

The stabilography evaluation was based on the most common practices used in research laboratories and the clinical environment (for a review see Duarte \& Freitas 2010; Paillard \& Noe 2015; Scoppa et al. 2013; Visser et al. 2008).

We evaluated the subjects' balance while standing still for $60 \mathrm{~s}$, in each of four different conditions: on a rigid surface with eyes open; on a rigid surface with eyes closed; on an unstable surface, a $6 \mathrm{~cm}$ height foam block (Balance Pad, Airex AG, Sins, Switzerland), with eyes open; on an unstable surface with eyes closed. Each condition was performed three times and the order of the conditions was randomized among subjects. The randomization was performed before the data collection by the examiner using a computerized random number generator. In all conditions, the subjects were required to stand, barefoot and as still as possible with their arms at their sides, and to look at a $5 \mathrm{~cm}$ round black target placed on the subject's eye-height on a wall $3 \mathrm{~m}$ ahead. For the trials where the eyes were kept closed, subjects were first instructed to look at the target with eyes open, find a stable and comfortable posture given the requirements, and 
102 close their eyes. A few seconds later, the data acquisition started. For all trials, the subject's feet

103 were placed with an angle of 20 degrees between them and their heels were kept $10 \mathrm{~cm}$ apart by

104 requesting the subjects to stand on lines marked on the top of the force platform (see Figure 1).

105 The trials were acquired in an empty $4.5 \times 2.8 \mathrm{~m}$ room with white walls and adequate

106 illumination (see Figure 1).

107

108

109

110

111

112

113
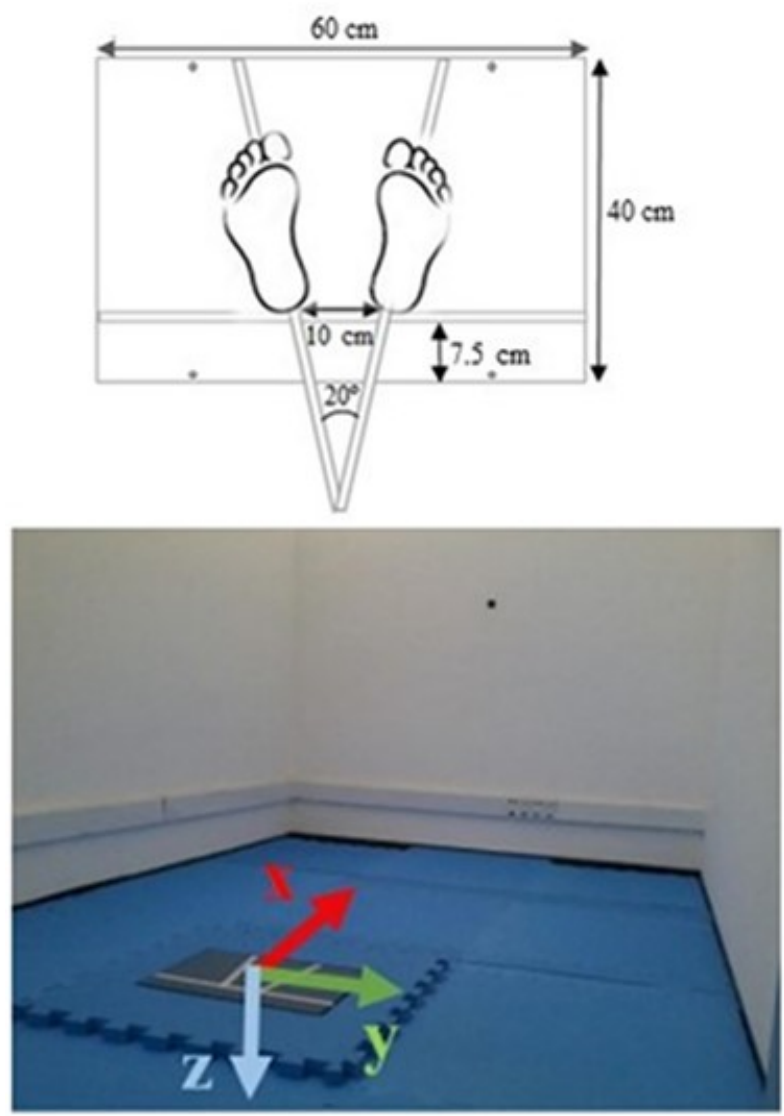

Figure 1. Top: Marks for the subject's feet placement on the force platform. Bottom: Data collection room for the stabilography (note the $5 \mathrm{~cm}$ black target at the wall $3 \mathrm{~m}$ ahead) and the force platform with its coordinate system convention.

\subsubsection{Protocol}

We followed the following procedure for the stabilography: 
114 1. The researcher explained to the subject about the data collection with the force plate. The

115 subject was also informed that during the data collection, he or she would be monitored,

116 there should not be any verbal communication during the trials, but he or she could interrupt

117 the data collection if desired and that assistance would be given if necessary;

118 2. The researcher recorded subject's name and identification number, the force platform was

119 zeroed and the subject was requested to stand on the force platform to record her or his

120 weight;

121 3. The researcher gave instructions on how the subject should stand on the force platform

122 according to the task (open or closed eyes, firm or form surface). The subject's feet was

123 positioned on the marks at the force platform (see Figure 1);

124 4. The researcher instructed the subject to maintain their arms along their body and to stand as 125 still as possible;

126 5. During the trials with eyes open, the subjects were told to fix their gaze on the round black 127 target placed on the wall ahead at the eye level;

128 6. During the trials with eyes closed, the subjects were told to fix their gaze at the target placed 129 ahead, close their eyes when they felt ready and only open them when the researcher 130 informed them of end of the trial;

131 7. The researcher started the data collection around $5 \mathrm{~s}$ later when the subject said he or she was 132 ready;

133 8. At the end of the trial, the subject was assisted to step from the force platform and he or she 134 could rest (and sit if desired) for about one minute before the next trial. 
1359 . If the subject was unable to complete a $60 \mathrm{~s}$ trial, the test was stopped and that trial was

136 immediately repeated up to two times if necessary. If, after the second attempt, the subject was still unable to complete the $60 \mathrm{~s}$ trial, that trial was considered missing.

\subsubsection{Data acquisition and processing}

The force platform signals were collected employing a $40 \mathrm{~cm}$ x $60 \mathrm{~cm}$ commercial force

force plate was factory-calibrated and presented an average COP accuracy of $0.02 \mathrm{~cm}$. However,

been shown that the installation procedure might have deteriorated the force platform calibration

of the measurement precision) was estimated as the standard-deviation value of the COP data when a $30 \mathrm{~kg}$ static load was placed on the force plate for $30 \mathrm{~s}$ and was equal to $0.005 \mathrm{~cm}$. The data acquisition was performed employing NetForce software (Version 3.5.3, AMTI, Watertown, MA, USA). The NetForce software outputs the calibrated forces and moments of forces (Fx, Fy, subsequent steps, reading these binary files, data processing, analysis and visualization, and exporting data to text files, were implemented in Python language using the SciPy Stack (https://www.scipy.org/) and are available as Jupyter Notebooks (http://jupyter.org/) in a GitHub repository (https://github.com/demotu/datasets). 
158 medio-lateral (y-positive is to the right) directions (see Figure 1) were calculated according to

159 the standard formulas (also described in the force platform manual):

160

$$
C O P_{x}=-\frac{M_{y}}{F_{z}}
$$

161

$$
\operatorname{COP}_{y}=\frac{M_{x}}{F_{z}}
$$

Note that the force platform data are expressed as forces and moments of forces in the

163 force platform co-ordinate system and they refer to the forces and moments of forces the subject

164 is applying on the force platform. This is the inverse of the ground reaction forces, where the

165 forces act on the subject (Newton's Third Law).

166

167

\subsection{Mini Balance Evaluation Systems Tests}

Each subject's balance was also evaluated by the Mini Balance Evaluation Systems Tests

(Mini-BESTest). The Mini-BESTest contains 14 items classified in four different domains of the

Organization; and Gait Stability; its maximum score is 28 points and each item varies from 0 (abnormal performance) to 2 (normal performance) points (Franchignoni et al. 2010). It has been shown that the Mini-BESTest, in general, presents excellent to good reliability and validity (Potter \& Brandfass 2015). The tasks in the Mini BESTest requiring the subjects' gaze fixed at a target were performed with the target placed $3 \mathrm{~m}$ ahead.

\section{$177 \quad 2.4 \quad$ Other evaluations}

178 In order to assess the subject's concern about falling, we applied the Short Falls Efficacy

179 Scale International (Short FES-I), which is a scale with seven items. The subjects rank their level 
180 of concern that they might fall if doing a hypothetical activity (Kempen et al. 2008). The Short

181 FES-I has excellent reliability and validity (Kempen et al. 2008). In order to characterize the

182 subject's practice of health-related physical activity, we applied the International Physical

183 Activity Questionnaire - Short Version (IPAQ-SV), which has eight questions about the time the 184 subject spends being physically active (Craig et al. 2003). The IPAQ-SV presents acceptable 185 reliability and validity (Craig et al. 2003). In order to characterize the subject's cognitive 186 functions, we applied the Trail Making Test (TMT), which instructs the subject to connect a set 187 of dots as quickly as possible (Reitan 1958). In general, the TMT has acceptable reliability and 188 validity (Poreh et al. 2012). These evaluations were conducted with personal interviews 189 performed in an empty $7.5 \times 5.7 \mathrm{~m}$ room with adequate illumination. We followed the 190 instructions of each evaluation test. For the IPAQ-SV, the last seven days prior to the interview 191 were considered. For the TMT, we followed the orientations by (Bowie \& Harvey 2006) to 192 administer parts A and B with the following details: the letter " $\mathrm{K}$ " was excluded in the evaluation 193 because it is less common to Brazilians and part B of the TMT was composed by 24 circles with 194 numbers from " 1 " to " 12 " and letters from "A" to "M".

\section{Results}

All the data is available at PhysioNet (DOI: 10.13026/C2WW2W) and at Figshare (DOI: 10.6084/m9.figshare.3394432). The data at PhysioNet (Goldberger et al. 2000) are stored in a binary format that can be read using for example, the WFDB software package or online using the physiobank ATM software and are made available under the ODC Public Domain Dedication and License v1.0 (http://opendatacommons.org/licenses/pddl/1.0/). The data at Figshare are stored in ASCII (text) format that can be downloaded as a single compressed file and are made available under the CC-BY license (https://creativecommons.org/licenses/by/4.0/). 

named BDSinfo (in two different formats, .txt or .xlsx) with meta data about each file, subject and the evaluation results. The data files and the BDSinfo.txt file are in ASCII format with tabseparated columns. The files with the force platform data have the following columns: Time, Fx, files are named as BDSxxxxx.txt, where BDS stands for the project's name, Balance Data Set, and $\operatorname{xxxxx}$ refers to the number of the trial; from 00001 to 01956 . For each subject there are 12 trials and the numbers in the file names are grouped in sets of 12, e.g., the first subject has files from 00001 to 00012 and the last subject (the 163rd), from 01945 to 01956 . A total of 26 files (trials) are missing for five subjects who were unable to complete the most challenging conditions.

The BDSinfo file contains meta data describing the conditions of the stabilography trials,

219 the information from the anamnesis, and the results of the qualitative evaluations. Because a subject has 12 files for the force platform data, there are 12 rows for each subject in this file. In these 12 rows, the only column that has rows with different values is the column identifying the trial (the file name). The content of all the other columns are simply repeated over the 12 rows. the meta data (the first word identifies the name of the column in the header): 01956). 
227 2. Subject: number of the subject (from 1 to 163 ).

228 3. Vision: visual condition (Open or Closed).

229 4. Surface: surface support condition (Firm or Foam).

230 5. Age: subject's age in years.

231 6. AgeGroup: age group (Young: Age $<60$; Old: Age $\geq 60$ ).

232 7. Gender: gender ( $F$ or $M)$.

233 8. Height: height in centimeters (measured with a calibrated stadiometer).

234 9. Weight: weight in kilograms (measured with a calibrated scale).

235 10. BMI: body mass index in $\mathrm{kg} / \mathrm{m}^{2}$.

236 11. FootLen: foot length in centimeters (average of the two feet, measured with a calibrated

237 paquimeter).

238 12. Nationality: country where the subject was born.

239 13. SkinColor: self-reported skin color.

240 14. Ystudy: years of regular study.

241 15. Footwear: most common type of footwear the subject wears daily.

242 16. Illness: whether the subject has any illness, as declared by themselves (Yes or No).

243 17. Illness2: type of illness of the subject ('No' if the subjects doesn't have any illness).

244 18. Nmedication: total number of medications the subject takes per day, if any.

245 19. Medication: name of the medication(s) the subject takes ('No' if the subject doesn't take any 246 medication).

247 20. Ortho-Prosthesis: whether the subject wears any type of orthosis or prosthesis, as declared 248 themselves (Yes or No). 
249 21. Ortho-Prosthesis2: name of the orthosis or prosthesis the subject wears ('No' if the subject

250 doesn't take any orthosis or prosthesis).

251 22. Disability: whether the subject has any deficit, as declared by themselves (Yes or No).

252 23. Disability2: name of the disability of the subject ('No' if the subject doesn't take any

253 disability).

254 24. Falls12m: how many non-intentional falls the subject had in the last 12 months, as declared

255 by themselves (from 0 to ...).

256 25. FES_1: answer for the first question of the Short Falls Efficacy Scale International test (FES-

257 I).

258 26. FES_2: answer for the second question of the FES-I.

259 27. FES_3: answer for the third question of the FES-I.

260 28. FES_4: answer for the fourth question of the FES-I.

261 29. FES_5: answer for the fifth question of the FES-I.

262 30. FES_6: answer for the sixth question of the FES-I.

263 31. FES_7: answer for the seventh question of the FES-I.

264 32. FES_T: answer for the total score question of the FES-I.

265 33. FES_S: answer for the scoring question of the FES-I, see

266 http://www.profane.eu.org/fesi.html.

267 34. IPAQ_1a: answer for the 1a question of the International Physical Activity Questionnaire 268 Short Version test (IPAQ).

269 35. IPAQ_1b: answer for the $1 \mathrm{~b}$ question of the IPAQ.

270 36. IPAQ_2a: answer for the 2a question of the IPAQ.

271 37. IPAQ_2b: answer for the 2b question of the IPAQ. 
272 38. IPAQ_3a: answer for the 3a question of the IPAQ.

273 39. IPAQ_3b: answer for the 3b question of the IPAQ.

274 40. IPAQ_4a: answer for the 4a question of the IPAQ.

275 41. IPAQ_4b: answer for the 4b question of the IPAQ.

276 42. IPAQ_S: score in the IPAQ (Low, Moderate, or High), see

277 https://sites.google.com/site/theipaq/.

278 43. TMT_timeA: time in seconds taken to complete part A of the Trail Making Test (TMT). We 279 didn't measure times longer than 5 minutes (for these cases we report a time of $300 \mathrm{~s}$ ).

280 44. TMT_errorsA: number of errors in part A of the TMT.

281 45. TMT_timeB: time in seconds taken to complete part B of the TMT. We did not measure 282 times longer than 5 minutes (for these cases we report a time of $300 \mathrm{~s}$ ).

283 46. TMT_errorsB: number of errors in part B of the TMT.

284 47. Best_1: score for the first task of the Mini Balance Evaluation Systems Test (Mini-BESTest).

285 48. Best_2: score for the second task of the Mini-BESTest.

286 49. Best_31: score for the third task (left side) of the Mini-BESTest.

287 50. Best_3r: score for the third task (right side) of the Mini-BESTest.

288 51. Best_4: score for the fourth task of the Mini-BESTest.

289 52. Best_5: score for the fifth task of the Mini-BESTest.

290 53. Best_6l: score for the sixth task (left side) of the Mini-BESTest.

291 54. Best_6r: score for the sixth task (right side) of the Mini-BESTest.

292 55. Best_7: score for the seventh task of the Mini-BESTest.

293 56. Best_8: score for the eighth task of the Mini-BESTest.

294 57. Best_9: score for the ninth task of the Mini-BESTest. 
295 58. Best_10: score for the tenth task of the Mini-BESTest.

296 59. Best_11: score for the eleventh task of the Mini-BESTest.

297 60. Best_12: score for the twelfth task of the Mini-BESTest.

298 61. Best_13: score for the thirteenth task of the Mini-BESTest.

299 62. Best_14: score for the fourteenth task of the Mini-BESTest.

300 63. Best_T: total score of the Mini-BESTest, see http://www.bestest.us/.

301 64. Date: date and time of the subject's evaluation (yyyy-mm-dd hh:mm:ss.sss 24-hour local 302 time format).

303

304

305

\subsection{Data exploration}

To exemplify how these data can be explored, shown in Figure 2 are histograms and 306 scatter plots for the variables: age, body mass, height, and BMI, and in Figure 3 are similar plots

307 for the variables: FES_T, Best_T, IPAQ_S, TMT_timeA, and TMT_timeB. A representative 308 example of the force platform data is shown in Figure 4 and plots for the variables: COP area, COP velocity, and COP mean frequency for each subject and standing condition are shown in Figure 5 (see Duarte 2015; Duarte \& Freitas 2010 for the algorithms to calculate these variables).

311 The programming scripts to generate these figures, as well as other examples on how to work 312 with these data, are available in a GitHub repository (https:/github.com/demotu/datasets). 

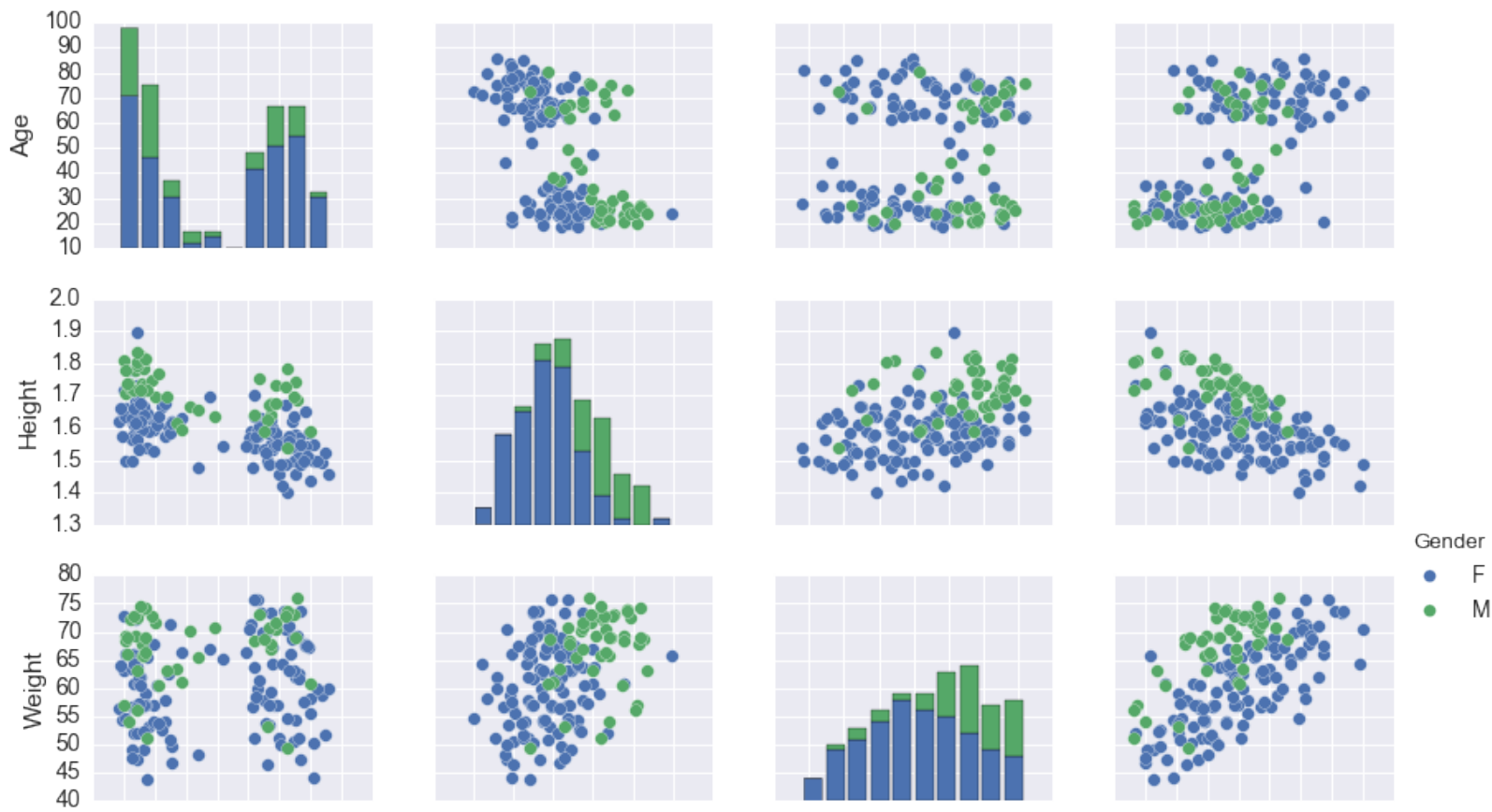

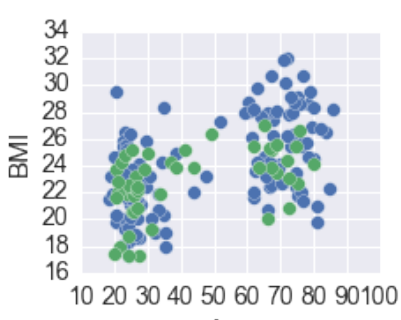

Age

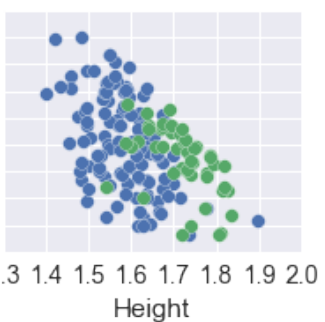

Height
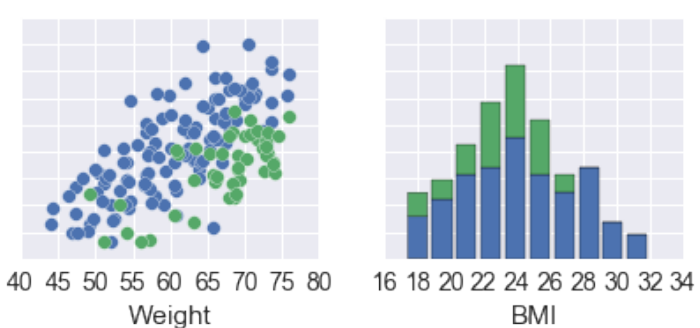

314 Figure 2. Histogram plots on the main diagonal and scatter plots outside the diagonal for the

315 following variables: age (years), body mass $(\mathrm{kg})$, height $(\mathrm{m})$, and BMI $\left(\mathrm{kg} / \mathrm{m}^{2}\right)$ for each subject.

316 These are color-coded by gender (116 females and 47 males). Note that, for the histograms on

317 the main diagonal, the actual vertical axes (the frequencies of observations occurring in certain

318 ranges of the variables shown on the bottom horizontal axes) are not shown. The name of the

319 variables plotted in each scatter plot outside the main diagonal are shown in the legends on the

320 left side and the bottom of the $4 \times 4$ panel. 

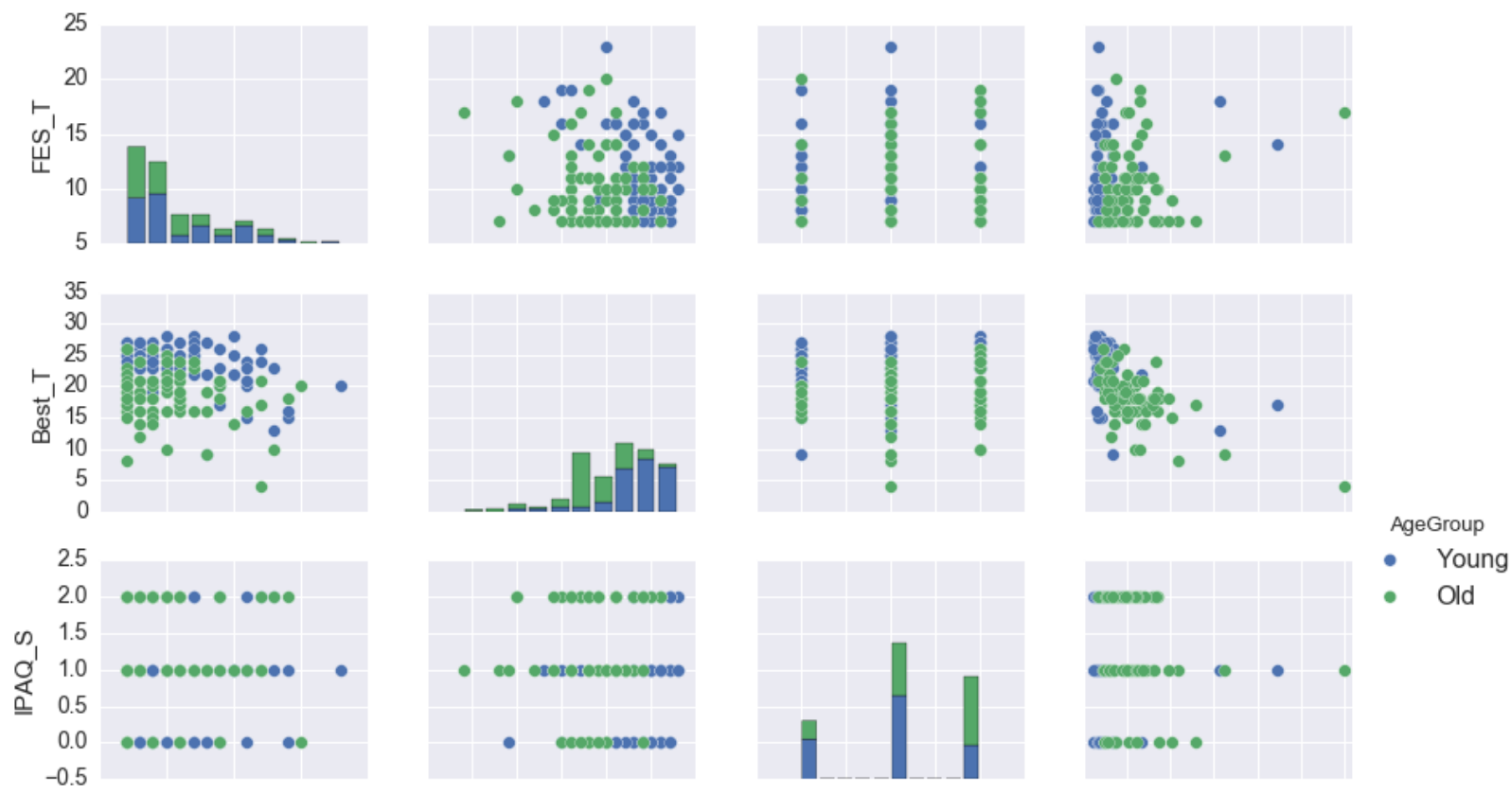

321
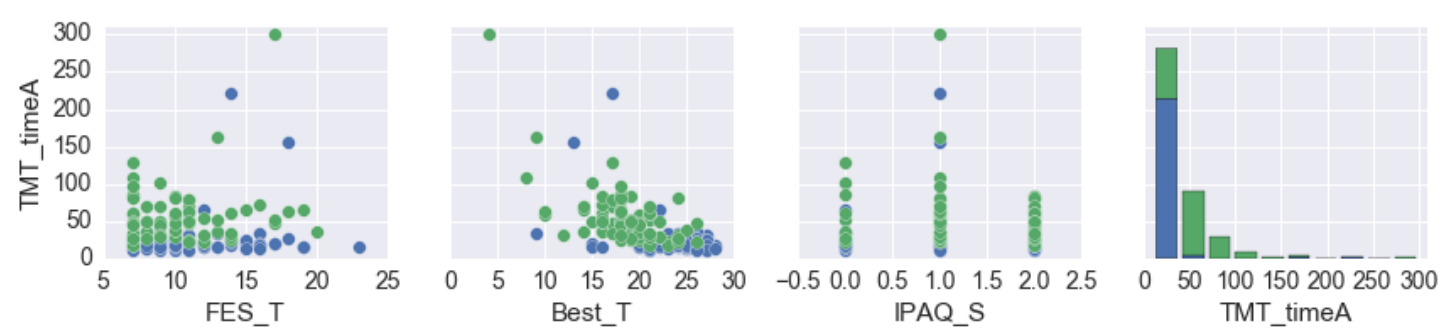

322 Figure 3. Histogram plots on the main diagonal and scatter plots outside the diagonal for the

323 following variables: FES_T, Best_T, IPAQ_S, and TMT_timeA. These are color-coded by age

324 group (87 young adults and 76 older adults). Note that, for the histograms on the main diagonal,

325 the actual vertical axes (the frequencies of observations occurring in certain ranges of the

326 variables shown on the bottom horizontal axes) are not shown. The name of the variables plotted

327 in each scatter plot outside the main diagonal are shown in the legends on the left side and the

328 bottom of the $4 \times 4$ panel. 


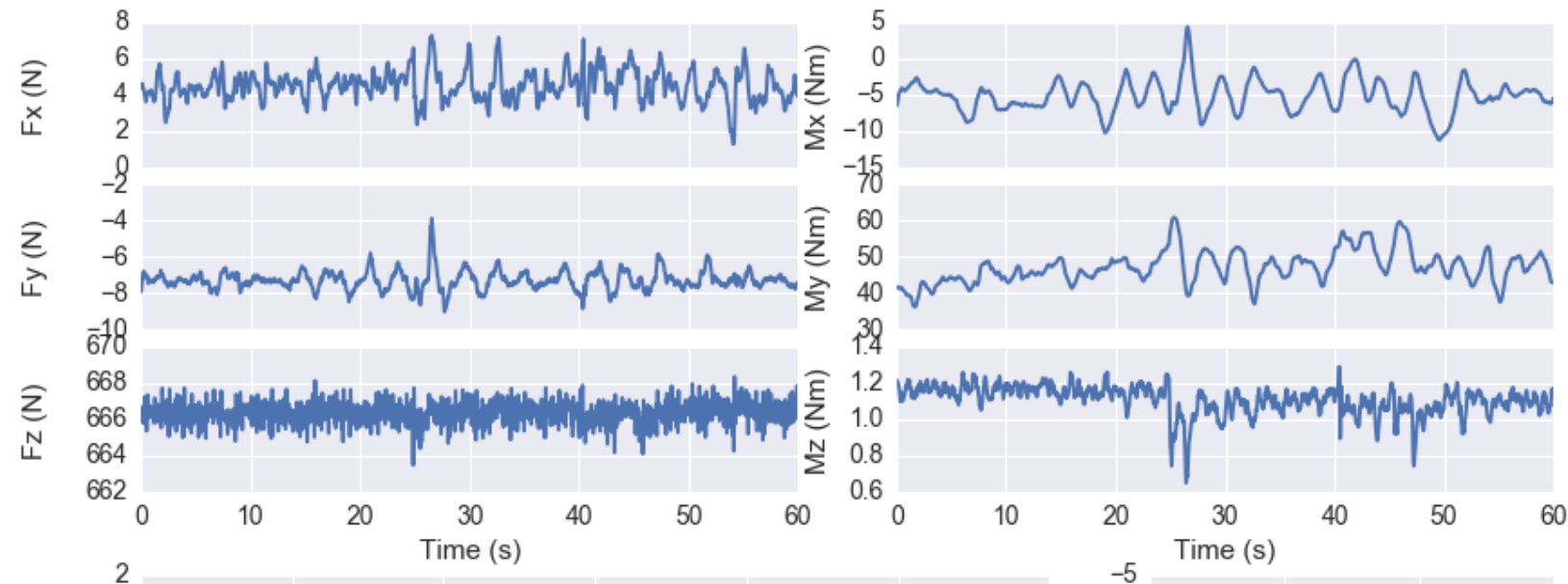

330 Figure 4: Top: exemplary forces and moments of forces at the horizontal and vertical anterior-

331 posterior (x), medio-lateral (y), and vertical (z) directions versus time. Bottom-left: center of

332 pressure (COP) displacements at the ap and $\mathrm{ml}$ directions versus time. Bottom-right: plot of COP

333 ap versus COP ml. Subject: 61-year-old male adult during quiet standing on a rigid surface with

334 open eyes. 

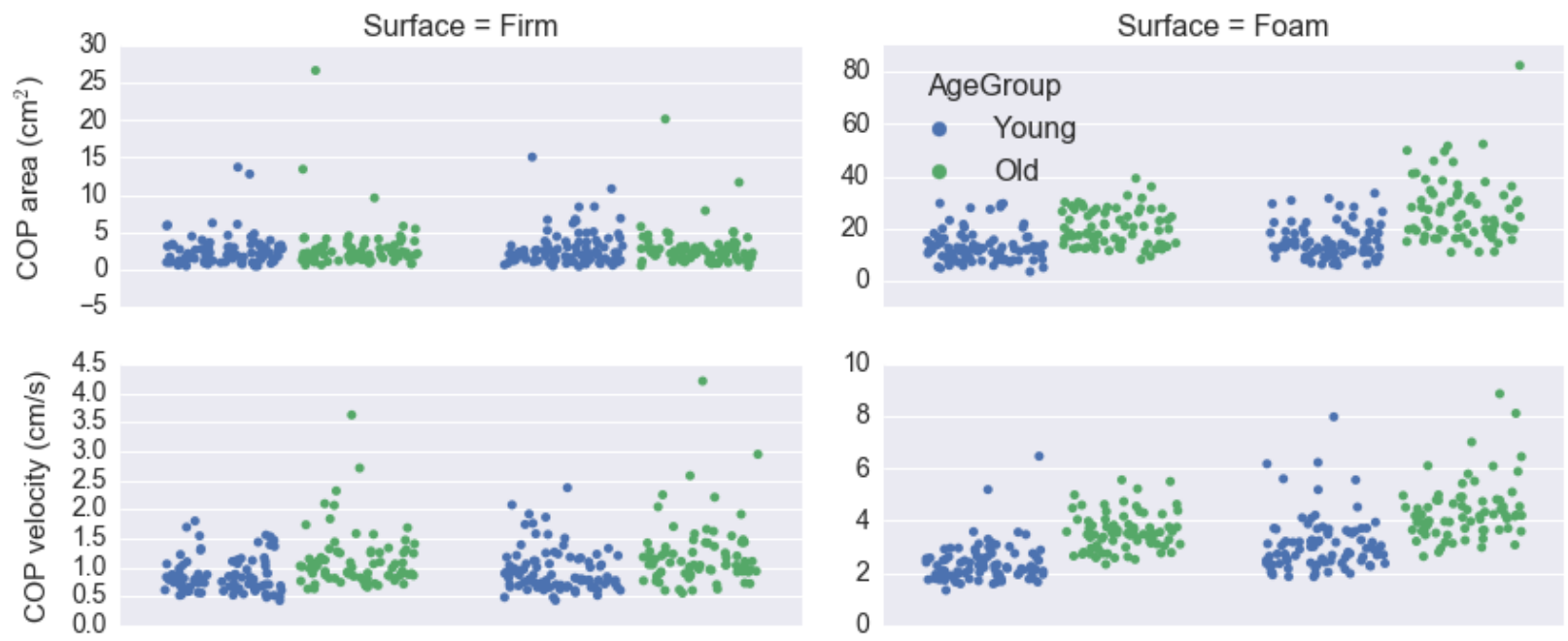

335

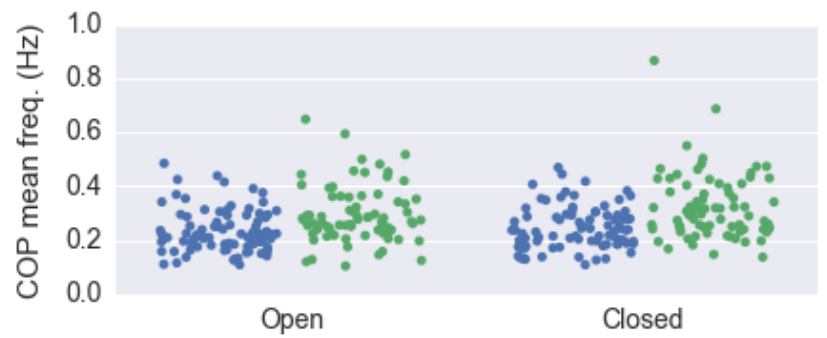

Vision

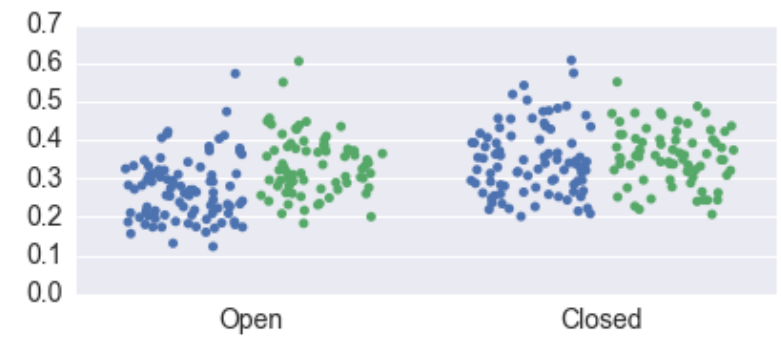

Vision

336 Figure 5. Plot of the median value across trials of the variables: COP area, resultant COP

337 velocity, and resultant COP mean frequency for each subject at the different visual and support

338 surface conditions color-coded by age group (87 young adults and 76 older adults).

\section{Discussion}

This study provides a publicly available data set with qualitative and quantitative

342 evaluations related to the balance of young and elderly adults (a total of 163 subjects). All the

343 data is available at PhysioNet (DOI: 10.13026/C2WW2W) and at Figshare (DOI:

10.6084/m9.figshare.3394432).

A limitation of this study is that we did not perform quantitative evaluations of the

346 subjects' health conditions, particularly of their motor and sensory integrity. However, through

347 the careful anamnesis we performed by interview, we were able to determine the history and 
348 current status of the subjects' health, and together with the different qualitative evaluations

349 performed, we believe the subjects are relatively well characterized. Nevertheless, the potential

350 user of this data set should bear this limitation in mind. We studied a convenience sample of

351 subjects who voluntarily accepted to participate in our study, which was conducted at a

352 university laboratory; as such, the sample might be unintentionally biased. Even though, we

353 classified 16 of the 163 investigated subjects as people with disabilities. If one is looking for

354 reference data on balance by healthy people, the data of these 16 subjects should be excluded.

355 One unintended characteristic of the data set is that the subjects can be roughly grouped in two

356 age groups: $20-40$ and 60-90 years old.

357 The Balance Data Set is the first public repository containing data of quantitative and

358 qualitative evaluations of human balance. Possible applications of this data set include: to test

359 new variables to describe the center of pressure displacement in methodological studies; to serve

360 as reference (normative) data for a new sample of subjects in a research of clinics context; for

361 training and education regarding the analysis of balance data, among others. Examples on how to

362 work with this data set are publicly available in a GitHub repository

363 (https://github.com/demotu/datasets).

3655 References

366 Bowie CR, and Harvey PD. 2006. Administration and interpretation of the Trail Making Test. Nat Protoc 1:2277-2281. 10.1038/nprot.2006.390

Cappello A, Bagala F, Cedraro A, and Chiari L. 2011. Non-linear re-calibration of force platforms. Gait \& Posture 33:724-726. 10.1016/j.gaitpost.2011.02.008 
country reliability and validity. Medicine and Science in Sports and Exercise 35:13811395. Doi 10.1249/01.Mss.0000078924.61453.Fb

374

375

376

377

378

379

Duarte M. 2015. Comments on "Ellipse area calculations and their applicability in posturography" (Schubert and Kirchner, vol.39, pages 518-522, 2014). Gait Posture 41:44-45. 10.1016/j.gaitpost.2014.08.008

Duarte M, and Freitas SM. 2010. Revision of posturography based on force plate for balance evaluation. Rev Bras Fisioter 14:183-192. S1413-35552010000300003 [pii]

Franchignoni F, Horak F, Godi M, Nardone A, and Giordano A. 2010. Using psychometric techniques to improve the Balance Evaluation Systems Test: the mini-BESTest. J Rehabil Med 42:323-331. 10.2340/16501977-0537

Goldberger AL, Amaral LA, Glass L, Hausdorff JM, Ivanov PC, Mark RG, Mietus JE, Moody GB, Peng CK, and Stanley HE. 2000. PhysioBank, PhysioToolkit, and PhysioNet: components of a new research resource for complex physiologic signals. Circulation 101:E215-220.

Kempen GIJM, Yardley L, van Haastregt JCM, Zijlstra GAR, Beyer N, Hauer K, and Todd C. 2008. The Short FES-I: a shortened version of the falls efficacy scale-international to assess fear of falling. Age Ageing 37:45-50. DOI 10.1093/ageing/afm157

Moore JK, Hnat SK, and van den Bogert AJ. 2015. An elaborate data set on human gait and the effect of mechanical perturbations. PeerJ 3:e918. 10.7717/peerj.918

Paillard T, and Noe F. 2015. Techniques and Methods for Testing the Postural Function in Healthy and Pathological Subjects. Biomed Res Int 2015:891390. 10.1155/2015/891390 
393 Poreh A, Miller, Dines, and Levin J. 2012. Decomposition of the Trail Making Test - Reliability 394 and Validity of a Computer Assisted Method for Data Collection. Archives of Assessment 395 Psychology 2.

396

397

398

399

400

401

402

403

404

405

406

407

408

409

410

411

412

413

414

415

Physiother 61:225. 10.1016/j.jphys.2015.04.002

Reitan RM. 1958. Validity of the Trail Making Test as an indicator of organic brain damage. Perceptual and Motor Skills 8:271-276.

Ruhe A, Fejer R, and Walker B. 2010. The test-retest reliability of centre of pressure measures in bipedal static task conditions--a systematic review of the literature. Gait \& Posture 32:436-445. 10.1016/j.gaitpost.2010.09.012

Samson M, and Crowe A. 1996. Intra-subject inconsistencies in quantitative assessments of body sway. Gait \& Posture 4:252-257. 10.1016/0966-6362(95)01050-5

Scoppa F, Capra R, Gallamini M, and Shiffer R. 2013. Clinical stabilometry standardization: basic definitions--acquisition interval--sampling frequency. Gait \& Posture 37:290-292. 10.1016/j.gaitpost.2012.07.009

Shumway-Cook A, and Woollacott MH. 2001. Motor control : theory and practical applications. Philadelphia: Lippincott Williams \& Wilkins.

Visser JE, Carpenter MG, van der Kooij H, and Bloem BR. 2008. The clinical utility of posturography. Clin Neurophysiol 119:2424-2436. 10.1016/j.clinph.2008.07.220

Yamamoto T, Smith CE, Suzuki Y, Kiyono K, Tanahashi T, Sakoda S, Morasso P, and Nomura T. 2015. Universal and individual characteristics of postural sway during quiet standing in healthy young adults. Physiol Rep 3. 10.14814/phy2.12329 